\title{
Separation-enrichment method for airborne disease spores based on microfluidic chip
}

\author{
Yafei Wang ${ }^{1,2}$, Xiaodong Zhang ${ }^{1,2}$, Ning Yang ${ }^{3}$, Guoxin $\mathrm{Ma}^{1,2}$, Xiaoxue $\mathrm{Du}^{1,2}$, \\ Hanping $\mathrm{MaO}^{1,2^{*}}$ \\ (1. School of Agricultural Engineering, Jiangsu University, Zhenjiang 212013, Jiansu, China; \\ 2. Key Laboratory of Modern Agricultural Equipment and Technology, Ministry of Education, Jiangsu University, \\ Zhenjiang 212013, Jiangsu, China; \\ 3. School of Electrical and Information Engineering, Jiangsu University, Zhenjiang 212013, Jiangsu, China)
}

\begin{abstract}
Airborne diseases are likely to cause crop yield reduction, which has aroused widespread concern. In this study, a two-stage separation-enrichment structure microfluidic chip with a compound field for separation and enrichment of the greenhouse crops airborne disease spores directly from gas flow was developed. The chip is mainly composed of three parts arc structure pretreatment channel, semicircular electrode structure and collection tank. COMSOL 5.1 software was used to simulate the designed microfluidic chip. $30 \mu \mathrm{m}$ particles were used to represent $P$. xanthii spores, $25 \mu \mathrm{m}$ particles were used to represent $P$. cubensis spores, and $16 \mu \mathrm{m}$ particles were used to represent $B$. cinerea spores. The simulation results showed that the separation and enrichment efficiency of $16 \mu \mathrm{m}$ particles, $25 \mu \mathrm{m}$ particles, and $30 \mu \mathrm{m}$ particles was $88 \%$, $91 \%$, and $94 \%$, respectively. The experimental verification results were observed under a microscope. The results showed that the separation and enrichment efficiency of B. cinereal spores, P. cubensis spores and P. xanthii spores was $75.7 \%, 83.8 \%$ and $89.4 \%$, respectively. As a result, the designed microfluidic chip can be used to separate and enrich the spores of airborne diseases of greenhouse crops, which can provide a basis for the research of real-time monitoring technology for greenhouse airborne diseases.
\end{abstract}

Keywords: greenhouse, crop disease, airborne spore, microfluidic chip

DOI: $10.25165 /$ j.ijabe.20211405.6375

Citation: Wang Y F, Zhang X D, Yang N, Ma G X, Du X X, Mao H P. Separation-enrichment method for airborne disease spores based on microfluidic chip. Int J Agric \& Biol Eng, 2021; 14(5): 199-205.

\section{Introduction}

With the continuously increasing global population, the demand for the 'vegetable basket' project has increased. At present, the vegetable industry is developing rapidly in China, with a greenhouse cultivation area of more than 4 million $\mathrm{hm}^{2}$, the largest in the world in terms of total area ${ }^{[1,2]}$. The environment in the greenhouse is conducive to the occurrence of airborne diseases, such as tomato gray mold (Botrytis cinerea), cucumber downy mildew (Pseudoperonospora cubensis), and cucumber powdery mildew (Podosphaera xanthii). When airborne diseases occur, with a yield loss of $20 \%-50 \%$, or even no harvest ${ }^{[3-5]}$. Therefore, accurate and timely monitoring of airborne disease spores is very important.

Received date: $2020-12-25 \quad$ Accepted date: 2021-07-07

Biographies: Yafei Wang, $\mathrm{PhD}$ candidate, research interest: intelligent agricultural equipment and technology, Email: wangyafei@126.com; Xiaodong Zhang, PhD, Associate Researcher, research interest: intelligent agricultural equipment and technology, Email: zxd700227@126.com; Ning Yang, PhD, Professor, research interest: intelligent agricultural equipment and technology, Email: yangning7410@163.com; Guoxin Ma, PhD candidate, research interest: intelligent agricultural equipment and technology, Email: Gavin_Ma123@ 163.com; Xiaoxue Du, PhD candidate, research interest: intelligent agricultural equipment and technology, Email: 2111916004@stmail.ujs.edu.cn.

* Corresponding author: Hanping Mao, $\mathrm{PhD}$, Professor, research interest: modern agricultural equipment and the Environmental control technology of facility agriculture. Department of agricultural equipment, Jiangsu University, Zhenjiang 212013, Jiangsu, China. Email: maohpujs@163.com.
At present, the detection methods of fungal spores mainly include image processing ${ }^{[6,7]}$, weighing method ${ }^{[8]}$, and polymerase chain reaction $(\mathrm{PCR})^{[9,10]}$. However, due to the presence of a large amount of impurity, the above methods are limited in the detection of fungal spores. Therefore, it is necessary to separate and enrich the spores to improve detection accuracy. Different types of crop fungal spores have different shapes and sizes. For example, B. cinerea spores are almost oval and have a size distribution of 11.4-26.7 $\mu \mathrm{m}, P$. cubensis spores are lemon shape and the size distribution is $21.1-39.8 \mu \mathrm{m}, P$. xanthii spores are cylindrical and the size distribution is $30.2 .1-39.5 \mu \mathrm{m}$. There are other objects defined as impurities in the actual detection environment, and their size is less than $10 \mu \mathrm{m}$.

Microfluidics is a science that studies precise control and manipulation of fluids through microchannels with dimensions of tens to hundreds of micrometers and the technology of manufacturing microminiaturized devices through which fluid flow is studied. Its numerous applications cover engineering, biological, biochemistry, nanotechnology, and many systems in which small volumes of fluids are processed to achieve high-throughput screening. Microfluidics has the potential to manipulate bioparticles aimed at focusing, trapping, separating, and sorting particles ${ }^{[11-13]}$. Wang et al. ${ }^{[14]}$ designed a microfluidic chip that can directly enrich airborne fungal spores from airflow. The chip includes three parts: half-wave pretreatment channel, inertial impactor and low-pressure collection chamber. Yang et al. ${ }^{[15]}$ designed a microfluidic chip to capture and detect rice disease spores and realize early monitoring of crop diseases. Although, 
the above research results can enrich and separate fungal spores in the air. However, fungal spores with similar shapes cannot be separated. Spores will be polarized under the action of a non-uniform electric field and move under the action of the electric field force. This phenomenon is called dielectrophoresis (DEP) ${ }^{[16]}$. DEP has been utilized widely for biomedical applications ${ }^{[17,18]}$. Over recent years, DEP is a non-invasive method that provides high-precision and high-efficiency analysis, which has attracted more and more attention. Zhang et al. ${ }^{[19]}$ designed a DEP microfluidic chip to achieve the separation of platelets and red blood cells. Zhang et al. ${ }^{[20]}$ presented a method based on DEP to rapidly separate and purify macrophages from micro-volume samples using a microfluidic device for liquid biopsy and point-of-care testing. Lee et al. ${ }^{[21]}$ designed a microfluidic device for continuous separation of nanoparticles by combining diffusiophoresis and electrophoresis to achieve the separation of nanoparticles having different sizes, in order to achieve high separation performance. Notwithstanding, many studies have been conducted on particles separation on microfluidic chip based on dielectrophoretic force. To the scrutiny of the current references, however, the separation of greenhouse crops airborne disease spore using DEP technique at a microfluidic device with the continuous flow has not yet been reported.

Hence, in this study, a two-stage separation-enrichment structure microfluidic chip with a compound field for separation and enrichment of the greenhouse crops airborne disease spores directly from gas flow was developed. It can separation-enrichment of greenhouse crops airborne disease spore and achieve timely monitoring of airborne diseases.

\section{Materials and methods}

\subsection{Theory and working principle}

The proposed microfluidic device's mathematical model is presented in this part; it is based on assumptions that are listed in the literature [22]. The model consists of the equations of motion, Navier-Stokes equation, continuity equation and electric field equation. The equations of motion are provided as follows ${ }^{[23]}$ :

$$
m_{e} \frac{\mathrm{d}^{2}}{\mathrm{~d} t^{2}} x_{e}=\sum F_{e x t}
$$

where, $t$ is the particle motion time, $\mathrm{s} ; m_{e}$ is the micro-scale entity's mass, $\mathrm{kg} ; x_{e}$ is the micro-scale entity's displacement, $\mathrm{m} ; F_{\text {ext }}$ is the external forces acting on the micro-scale entity. The inertial force associated with the micro-scale entity is presented on the left-hand side of Equation (1). The external forces to which a micro-scale entity is subjected are that due to gravity, drag, buoyancy, and DEP.

DEP refers to the movement of a polarizable particle in response to the interactions between the induced dipole moment of the particle and the spatially non-uniform electric field ${ }^{[24]}$. When a particle of radius $r$ suspended in the medium is subjected to a non-uniform electric field $E$, the averaged DEP force $\left(F_{\mathrm{DEP}}\right)$ acting on the spores is defined as ${ }^{[25,26]}$

$$
F_{\mathrm{DEP}}=2 \pi \varepsilon_{m} r^{3} \operatorname{Re}\left[K^{*}(\omega)\right] \nabla\left(|E|^{2}\right)
$$

where, $r$ is the radius of the particle, $\mu \mathrm{m} ; \varepsilon_{m}$ is the permittivity of the medium; $\nabla$ is the gradient operator. $\omega=2 \pi f$ is the angular frequency. $\operatorname{Re}\left[K^{*}(\omega)\right]$ is the real part of the complex Claussius-Mossotti (CM) factor ${ }^{[27,28]}$, which is given by

$$
\begin{aligned}
\varepsilon^{*} & =\varepsilon-\frac{j \sigma}{\omega} \\
\sigma^{*} & =\sigma+j \omega \varepsilon_{0} \varepsilon
\end{aligned}
$$

$$
\begin{aligned}
& K^{*}(\omega)=\frac{\varepsilon_{p}^{*}-\varepsilon_{m}^{*}}{\varepsilon_{p}^{*}+2 \varepsilon_{m}^{*}}=\frac{\left(\varepsilon_{p}-\varepsilon_{m}\right)+j \frac{\sigma_{m}-\sigma_{p}}{\omega}}{\left(\varepsilon_{p}+2 \varepsilon_{m}\right)-j \frac{2 \sigma_{m}+\sigma_{p}}{\omega}} \\
& \operatorname{Re}\left[K^{*}(\omega)\right]=\operatorname{Re}\left(\frac{\varepsilon_{p}^{*}-\varepsilon_{m}^{*}}{\varepsilon_{p}^{*}+2 \varepsilon_{m}^{*}}\right)= \\
& \frac{\omega^{2}\left(\varepsilon_{p}-\varepsilon_{m}\right)\left(\varepsilon_{p}+2 \varepsilon_{m}\right)+\left(\sigma_{p}-\sigma_{m}\right)\left(2 \sigma_{m}+\sigma_{p}\right)}{\omega^{2}\left(\varepsilon_{p}+2 \varepsilon_{m}\right)^{2}+\left(2 \sigma_{m}+\sigma_{p}\right)^{2}}
\end{aligned}
$$

where, $j$ is the imaginary part of the function; $\varepsilon$ and $\sigma$ is the absolute permittivity and conductivity, respectively. The subscripts $p$ and $m$ denote the particle and the medium, respectively. $\varepsilon_{0}$ is the vacuum dielectric constant $\left(\varepsilon_{0} \approx 8.85 \times 10^{-12} \mathrm{~F} / \mathrm{m}\right)$. Equation (2) shows that:

1) The $F_{\mathrm{DEP}}$ received by the microparticle is proportional to the third power of the radius of the microparticle. Therefore, microparticles of different sizes will be subjected to $F_{\mathrm{DEP}}$ of different sizes due to their different sizes.

2) The size of the $F_{\mathrm{DEP}}$ on the microparticles has a positive correlation to the dielectric constant of the air medium.

3) $F_{\text {DEP }}$ received by the microparticles is proportional to the square of the electric field intensity gradient. Therefore, by designing the shape of the electrode to increase the gradient of the electric field intensity, the dielectrophoretic force received by the microparticles can be increased.

4) Because the sign of the real part of the $\mathrm{CM}$ factor determines the direction of the $F_{\mathrm{DEP}}$. Therefore, the direction of the microparticles subjected to the dielectrophoretic force depends on the electrical conductivity, dielectric constant and alternating current frequency of the microparticles and the air medium. Because the dielectric constant of air is equal to 1 , and the conductivity is equal to $0, \operatorname{Re}\left[K^{*}(\omega)\right]$ is approximately equal to 1 . Therefore, the microparticles will be subjected to the positive DEP force ( $\mathrm{pDEP}$ ), that is, the microparticles will move to the area with high electric field intensity.

The analysis of the $F_{\mathrm{DEP}}$ on the microparticles in the $\mathrm{AC}$ electric field shows that the microparticles will receive the pDEP when they move in the gas. When different types of microparticles are separated by $F_{\mathrm{DEP}}$, the separation can be achieved according to the size of the microparticles and the magnitude of the dielectrophoretic force received.

When manipulating the microparticles in the microfluid, due to the low volume concentration of the microparticles, and the size of the microparticles is much smaller than the characteristic size of the chip, the force between the microparticles and the reaction force of the microparticles on the flow field can be ignored. Therefore, the force of the microparticles in the microfluid by the fluid mainly includes stokes force, Basset force, buoyancy force and additional mass force. Because the microparticles in the microfluid are basically suspended, the buoyancy is almost equal to gravity. Therefore, according to the actual situation, the influence of gravity and buoyancy is ignored, and the influence of the inertial force of the movement of the microparticle on the manipulation of the microparticle is ignored. Therefore, only the stokes force is considered in the fluid force on the microparticles.

In this study, a micropump was used to push the microfluidic gas and microfluid into the microchannel through pressure drive. It is assumed that the speed of fluid flow is stable and the speed of microparticles is relatively small. Particles in a pressure drive flow experience a hydrodynamic drag force expressed as,

$$
F_{\mathrm{st}}=6 \pi \mu r\left(V_{m}-V_{p}\right)
$$


where, $\mu$ is the dynamic viscosity of the fluid. $V_{\mathrm{m}}(\mathrm{m} / \mathrm{s})$ and $V_{\mathrm{p}}$ $(\mathrm{m} / \mathrm{s})$ refer to the velocity of the medium and particle, respectively. The direction of the vector difference between the velocity of the microfluid and the velocity of the microparticle determines the direction in which the microparticle receives the Stokes force $F_{\text {st }}$.

In this study, a single shell model was used instead of disease spores $^{[19]}$. First, the simplest modeling is the single-shell model ${ }^{[29]}$, whose effective dielectric constant is $\varepsilon_{\text {spore. }}^{*}$.

$$
\varepsilon_{\text {spore }}^{*}=\frac{C_{\mathrm{mem}}^{*} r \varepsilon_{c}^{*}}{C_{\mathrm{mem}}^{*} r+\varepsilon_{c}^{*}}
$$

where, the $C_{\text {mem }}^{*}$ and $\varepsilon_{c}^{*}$ respectively denote the complex mem-brane capacitance and the complex permittivity of the spore, given by

$$
\begin{aligned}
C_{\mathrm{mem}}^{*} & =C_{\mathrm{mem}}+\frac{g_{\mathrm{mem}}}{j \omega} \\
\varepsilon_{c}^{*} & =\varepsilon_{c}-j \frac{\sigma_{c}}{\omega}
\end{aligned}
$$

where, $C_{\text {mem }}, g_{\text {mem }}, \varepsilon_{c}$ and $\sigma_{c}$ are the membrane capacitance and conductance per unit area, the spore permittivity and conductivity, respectively. The typical values of these parameters can be found in early publications ${ }^{[30]}$.

\subsection{Two-dimensional simulation model of chip}

In order to achieve efficient separation and enrichment of greenhouse crops airborne disease spore. Design a two-stage separation-enrichment structure microfluidic chip with the compound field. The two-dimensional diagram of the

microfluidic chip is shown in Figure 1. The first structure includes particles entrance, channel 1, arc structure and channel 2. The second structure includes channel 3, channel 4, channel 5, semicircular electrode structure, collection tank and particle exit. $R_{1}$ is the radius of arc channels. $R_{2}$ is the radius of the collection tank. $\quad R_{3}$ is the radius of the semicircular electrode. $\theta_{1}$ and $\theta_{2}$ are the separation angles of the particle channels, respectively. The width of channel 1 was set at $L_{1}=2000 \mu \mathrm{m}$, length of channel $1=$ $11000 \mu \mathrm{m}, R_{1}=1000 \mu \mathrm{m}$, width of arc channel $1=2000 \mu \mathrm{m}$, length of channel $2=4700 \mu \mathrm{m}$, the width of channel 2 was set $L_{2}=$ $1500 \mu \mathrm{m}$, length of channel $3 \mathrm{a}$ and channel $3 \mathrm{~b}=9000 \mu \mathrm{m}$, the width of channel $3 \mathrm{a}$ and channel $3 \mathrm{~b}$ were set $L_{3}=2000 \mu \mathrm{m}$, length of channel $4 \mathrm{a}$, channel $4 \mathrm{~b}$, channel $4 \mathrm{c}$ and channel $4 \mathrm{~d}=2300 \mu \mathrm{m}$, the width of channel $4 \mathrm{a}$, channel $4 \mathrm{~b}$, channel $4 \mathrm{c}$ and channel $4 \mathrm{~d}$ were set $L_{4}=1500 \mu \mathrm{m}$, length of channel 5a, channel 5b, channel $5 \mathrm{c}$ and channel $5 \mathrm{~d}=2000 \mu \mathrm{m}$, the width of channel $5 \mathrm{a}$, channel $5 \mathrm{~b}$, channel $5 \mathrm{c}$ and channel $5 \mathrm{~d}$ were set $L_{5}=650 \mu \mathrm{m}, R_{2}=1000 \mu \mathrm{m}$. The height of the microchannel is $100 \mu \mathrm{m}$. The range of $\theta_{1}$ and $\theta_{2}$ is $10^{\circ}-40^{\circ}$.

In this study, the finite element analysis software COMSOL Multiphysics 5.1 was used for simulation analysis. The laminar flow module, AC/DC module, and particle trajectory tracking module in the software were used to simulate the separation process. Particle Trajectory Tracking Module coupling the influence of the flow field and electric field on the particle trajectory was used to calculate the trajectory of the particles in the channel.

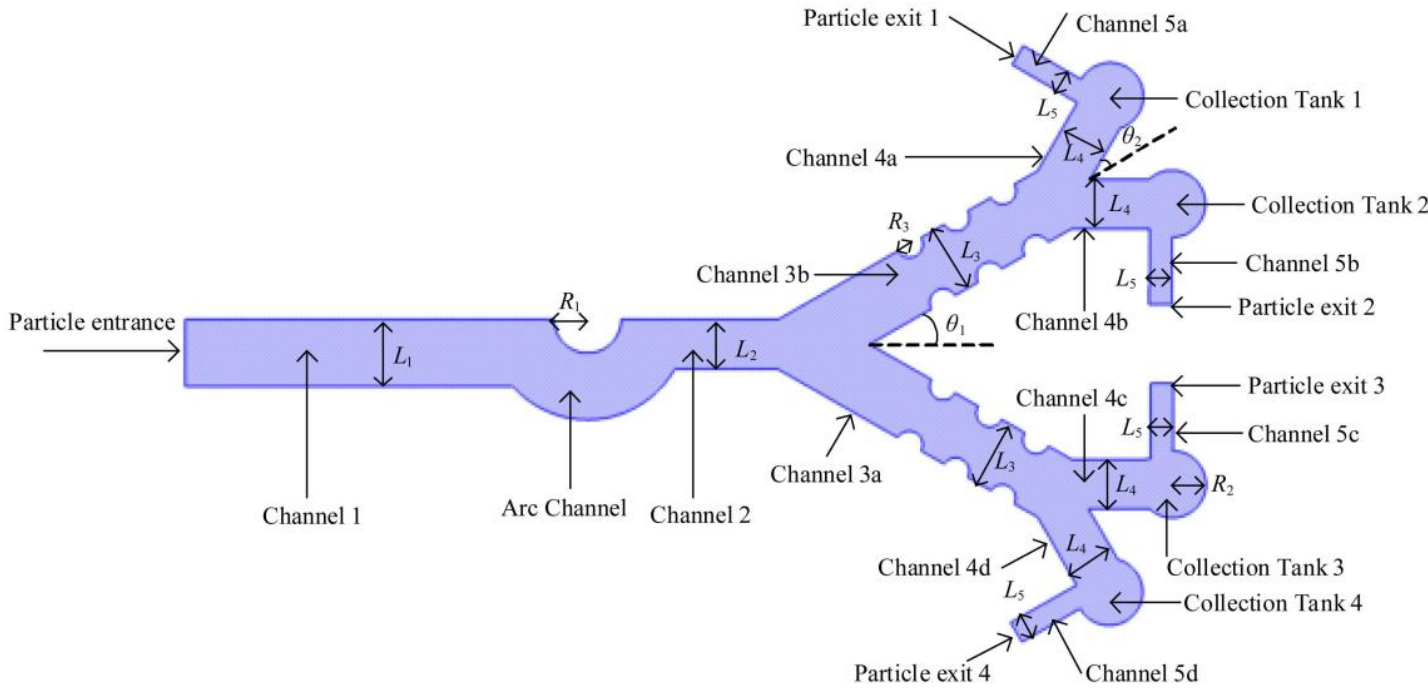

Note: $L_{1}$ is the width of channel $1 ; L_{2}$ is the width of channel $2 ; L_{3}$ is the width of channel $3 \mathrm{a}$ and channel $3 \mathrm{~b} ; L_{4}$ is the width of channel $4 \mathrm{a}$, channel $4 \mathrm{~b}$, channel $4 \mathrm{c}$ and channel $4 \mathrm{~d} ; L_{5}$ is the width of channel $5 \mathrm{a}$, channel $5 \mathrm{~b}$, channel $5 \mathrm{c}$ and channel $5 \mathrm{~d} ; R_{1}$ and $R_{3}$ are the radii of arc; $R_{2}$ is the radius of collection tank 1 , collection tank 2 , collection tank 3 and collection tank 4 .

Figure 1 Two-dimensional diagram of microfluidic chip

\subsection{Numerical simulation methods and boundary conditions}

The flow field and electric field distribution in the channel were solved in the laminar flow module and the AC/DC module. Laminar flow corresponds to the flow with small $\operatorname{Re}(\operatorname{Re}<1)$, the viscosity term of the Navier-Stokes equation dominates and the inertia term can be ignored. At this point, Conditions are 1) The channel wall is a non-slip boundary; 2) Flow rates of the inlet is $16 \mathrm{~mL} / \mathrm{min}$; 3) Pressure at the outlet is set to be atmospheric pressure, that is $p=0$. The flow was assumed to be steady, two-dimensional axisymmetric, and incompressible. The temperature was $293.15 \mathrm{~K}$ and the applied pressure was $101.3 \mathrm{kPa}$. The wall was set to adhere. The particle density was set at $1050 \mathrm{~kg} / \mathrm{m}^{3}$. And 100 particles were released from the particle entrance.
A particle tracking module was used in COMSOL 5.1. The dielectrophoretic force and the Stokes force applied to the particles were obtained by solving the electric field and the flow field, respectively. The governing equations for $F_{\mathrm{DEP}}$ are given in Equations (2)-(6). At the same time, the single-shell model was used for spore particles, and the governing equation was given by Equation (8). At this point, the corresponding boundary conditions are 1) The channel wall condition is rebound; 2) The initial velocity of the inlet fluid is based on the velocity field in the laminar flow; 3) The drag force follows Stokes' law. Using post-processing in COMSOL 5.1, select the global calculation in the derived value. Data set select the particles to be released, and the expression is the total number of particles in the selection. Count the particles collected in the collection tank area. The 
enrichment efficiency of particles was obtained.

\subsection{Spore sample preparation}

$B$. cinerea spores, $P$. cubensis spores, and $P$. xanthii spores were collected from the plants' leaves in the Greenhouse of the Key Laboratory of Modern Agricultural Equipment and Technology, Ministry of Education, Jiangsu University, Zhenjiang, Jiangsu, China. Disease spores were inoculated on PDA medium. Propagation was performed on plant leaves when necessary. The morphology of spores was observed under an ultra-deep, three-dimensional microscope (VHX-900F, made by KEYENCE Co., Osaka, Japan $)^{[31]}$. The measurement results are shown in Table 1.

Table 1 Spore size of three greenhouse crops airborne disease

\begin{tabular}{ll}
\hline \multicolumn{1}{c}{ Species } & \multicolumn{1}{c}{ Spore $\operatorname{size} / \mu \mathrm{m} \times \mu \mathrm{m}$} \\
\hline P. xanthii & $35.4(30.2-39.5) \times 14.2(7.3-22.2)$ \\
P. cubensis & $30.6(21.1-39.8) \times 20.5(13.8-23.6)$ \\
B. cinerea & $19.3(11.4-26.7) \times 11.7(8.3-14.5)$ \\
\hline
\end{tabular}

The spores in the air are mainly ungerminated spores $^{[32]}$. Therefore, $30 \mu \mathrm{m}$ particles were used to represent $P$. xanthii spores, $25 \mu \mathrm{m}$ particles were used to represent $P$. cubensis spores, and $16 \mu \mathrm{m}$ particles were used to represent $B$. cinerea spores. less than $10 \mu \mathrm{m}$ particles were used to represent impurities in the environment.

\subsection{Evaluation of airborne disease spore collection efficiency}

The collection efficiency of particles can be defined as ${ }^{[33]}$

$$
\eta=\frac{\eta_{i}}{\eta_{i}+\eta_{j}} \times 100 \%
$$

where, $\eta_{i}$ is the number of given particle size spores that pass through the chip and enter the collection tank; $\eta_{j}$ is the sum of the number of given particle size spores in the wall of the microchannel in the chip, at each outlet position and in other collection tanks.

\section{Results and discussion}

\subsection{Particle motion simulation}

When the particles pass through channel 1 and the arc-shaped channel 1, the force acting on the particles is the same, but the direction of the force is different. The original separation of particles can be achieved. More than $25 \mu \mathrm{m}$ particles enter channel $3 \mathrm{a}$ due to their larger velocity. When the gas passes through the separation angle $\theta_{1}$, the direction and velocity of the gas flow will change in channel $3 \mathrm{a}$ and channel $3 \mathrm{~b}$. The speed measured inside the channel is greater than the speed outside. The particles in channel $3 a$ and channel $3 b$ are further separated under different gas velocities, gas directions, $\theta_{2}$ and $F_{\text {DEP. }}$. The $30 \mu \mathrm{m}$ particles and $16 \mu \mathrm{m}$ particles enter collection tank 4 and collection tank 1 , respectively. The $25 \mu \mathrm{m}$ particles and less than $10 \mu \mathrm{m}$ particles enter collection tank 3 and collection tank 2, respectively. In order to achieve this goal, the values of $\theta_{1}$ and $\theta_{2}$ need to be numerically simulated. When $\theta_{1}$ and $\theta_{2}$ take different values, the enrichment results of particles are shown in Figure 2.

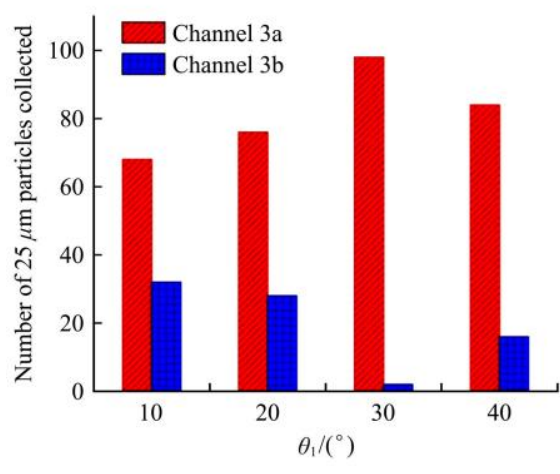

a. Effect of angle $\theta_{1}$ on $25 \mu \mathrm{m}$ particle separation and enrichment

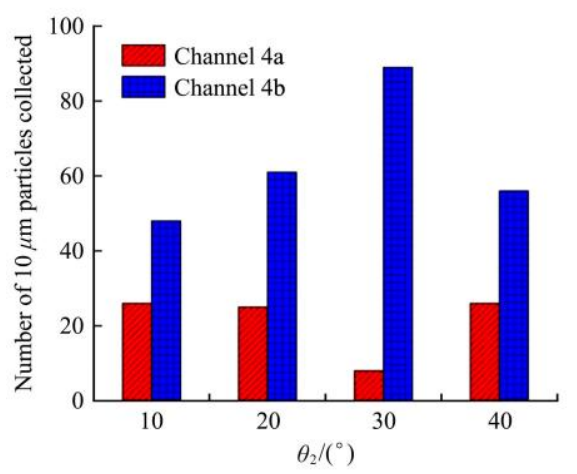

d. Effect of angle $\theta_{2}$ on $10 \mu \mathrm{m}$ particle separation and enrichment

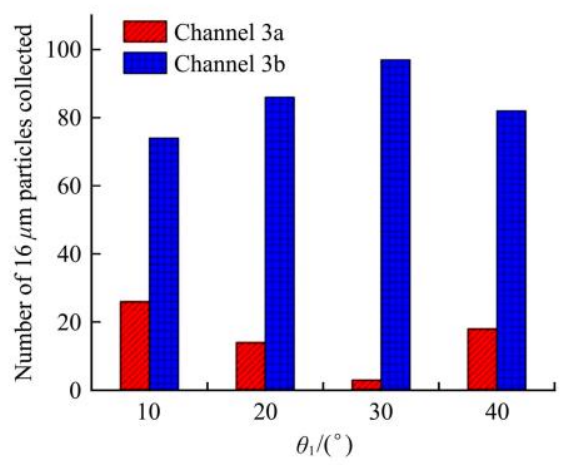

b. Effect of angle $\theta_{1}$ on $16 \mu \mathrm{m}$ particle separation and enrichment

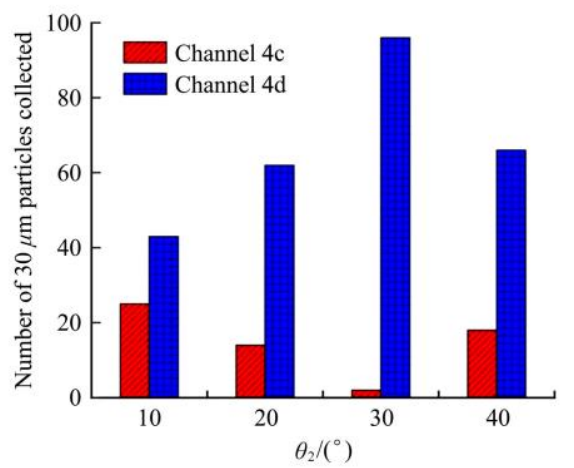

e. Effect of angle $\theta_{2}$ on $30 \mu \mathrm{m}$ particle separation and enrichment

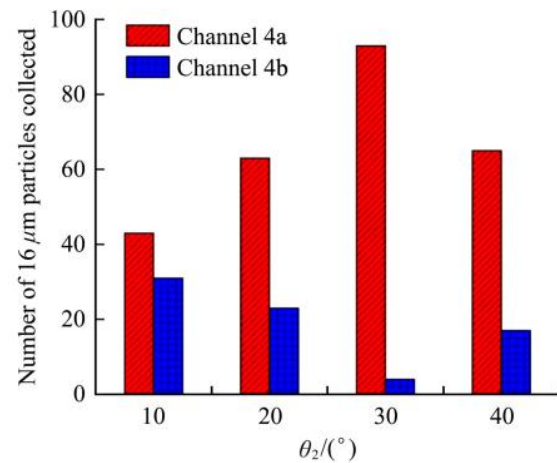

c. Effect of angle $\theta_{2}$ on $16 \mu \mathrm{m}$ particle separation and enrichment

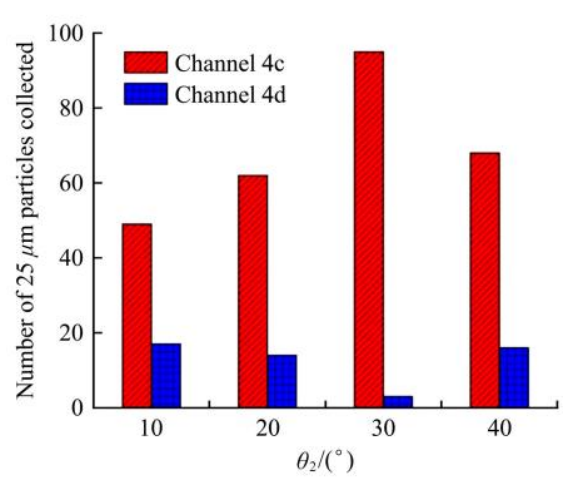

f. Effect of angle $\theta_{2}$ on $25 \mu \mathrm{m}$ particle separation and enrichment

Figure 2 Effects of angle $\theta_{1}$ and $\theta_{2}$ on particle separation and enrichment

It can be seen from Figure 2 that when $\theta_{1}$ and $\theta_{2}$ are $30^{\circ}$, the separation and enrichment efficiency of particles is relatively high. Therefore, set $\theta_{1}$ and $\theta_{2}$ at $30^{\circ}$ for the next step of numerical simulation in this study. Because the dielectric constant of air is equal to 1 , and the conductivity is equal to $0^{[34]}$. It can be seen from Equation (6), $\operatorname{Re}\left[K^{*}(\omega)\right]$ is approximately equal to 1 . According to
Equation (2), $F_{\mathrm{DEP}}$ on the particle is related to the characteristics of the particle and the electrode voltage. The electrode voltage was set to $120 \mathrm{~V}$, at the frequency of $10^{6} \mathrm{~Hz}^{[35]}$. Figures $3 \mathrm{a}-3 \mathrm{~d}$ are the simulation results of four kinds of particles. Figure $3 e$ is the intensity of pressure distribution in the microfluidic of the chip. Figure $3 \mathrm{f}$ is the velocity distribution in the microchannel of the chip. 


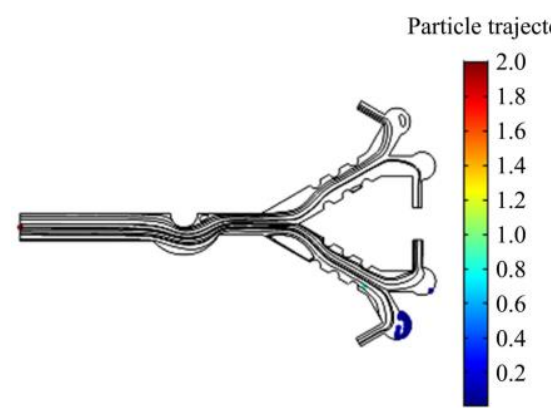

a. $30 \mu \mathrm{m}$ particle trajectory

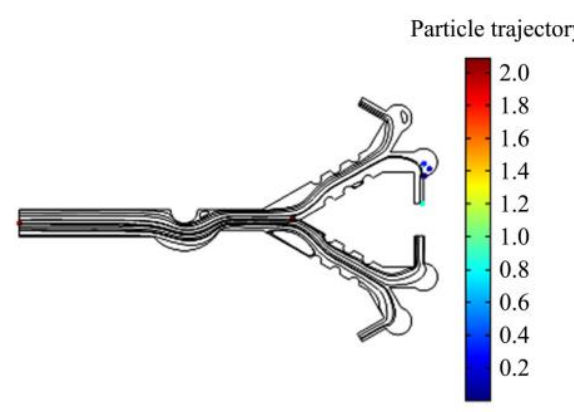

d. $10 \mu \mathrm{m}$ particle trajectory

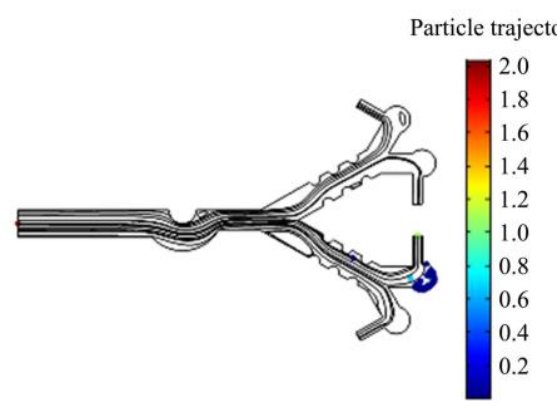

b. $25 \mu \mathrm{m}$ particle trajectory

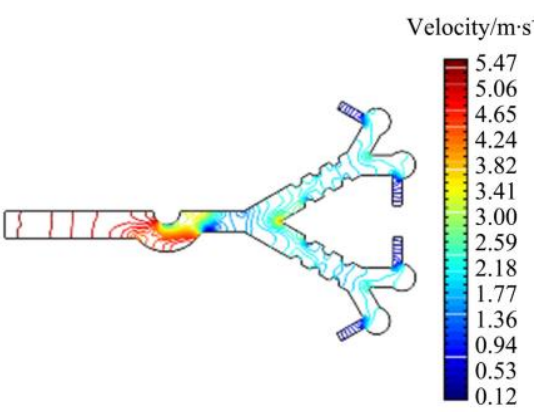

e. Velocity distribution

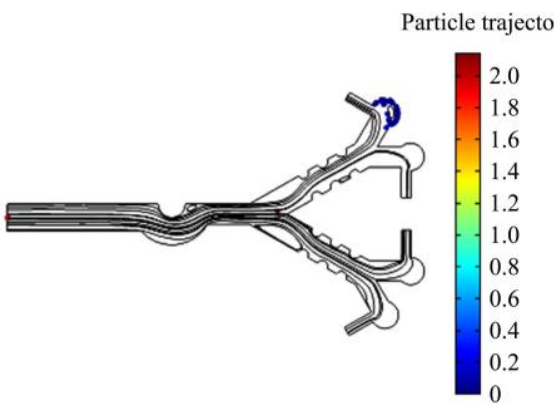

c. $16 \mu \mathrm{m}$ particle trajectory

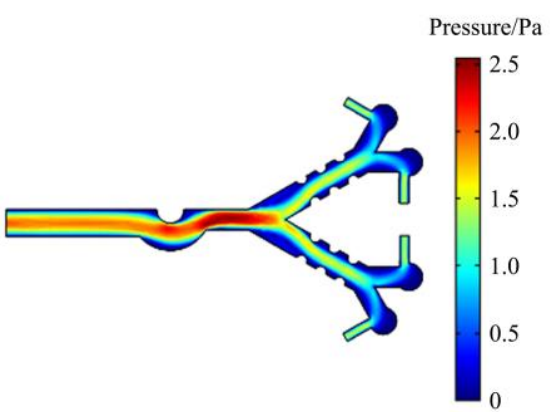

f. Pressure distribution

Figure 3 Simulation results of microfluidic chip

As shown in Figure 3, gas enters the microchannel from the particles entrance and obtains a horizontal to right initial velocity $^{[14,15]}$. The particles enter channel $3 \mathrm{a}$ and channel $3 \mathrm{~b}$ under the action of centrifugal force. When the arc channel is added, the arc channel has a coupling effect with the $\theta_{1}$ angle turn, which increases the centrifugal force of the particles, so that it can enter channel 3a and channel $3 \mathrm{~b}$. The density of all the particles is the same, so the larger the diameter of the particles, the greater the centrifugal force. More than $25 \mu \mathrm{m}$ particles can enter channel 3a, while less than $16 \mu \mathrm{m}$ particles can enter channel $3 \mathrm{~b}$. When the gas passes through the separation angle $\theta_{1}$, the direction and velocity of the gas flow will change in channel $3 \mathrm{a}$ and channel $3 \mathrm{~b}$. The particles in channel $3 a$ and channel $3 b$ were further separated under different gas velocities, gas directions, the separation angle $\theta_{2}$ and $F_{\mathrm{DEP}}$. The $16 \mu \mathrm{m}$ particles and $30 \mu \mathrm{m}$ particles enter collection tank 1 and collection tank 4 , respectively. The $10 \mu \mathrm{m}$ particles and less than $25 \mu \mathrm{m}$ particles enter collection tank 2 and collection tank 3 , respectively. In order to calculate the enrichment results of the particles, four kinds of particles were released from the particles entrance, respectively. The statistical results of particle enrichment are shown in Figure 4.

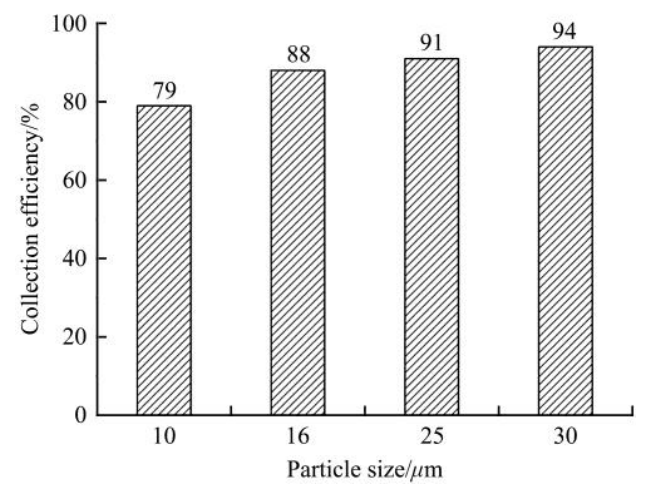

Figure 4 Statistical results of particle enrichment

As can be seen from Figure 4, separation and enrichment efficiency of $10 \mu \mathrm{m}$ particles, $16 \mu \mathrm{m}$ particles, $25 \mu \mathrm{m}$ particles and $30 \mu \mathrm{m}$ particles was $79 \%, 88 \%, 91 \%$ and $94 \%$, respectively. The enrichment efficiency of $30 \mu \mathrm{m}$ particles was highest. The enrichment efficiency of $10 \mu \mathrm{m}$ particles was low. Because $10 \mu \mathrm{m}$ particles have a smaller diameter and smaller inertia when the density is constant, and they flow out from the particle exit 2 as the gas flows out ${ }^{[36]}$.

\subsection{Experimental verification}

The schematic diagram of the experimental platform is shown in Figure 5, including the aerosol generator, the microfluidic device, the dielectrophoretic force generation system and the measurement system.

Airborne disease spore suspension from greenhouse crops was placed in aerosol generators to maintain the integrity and biological activity of spores at low pressure. The compressed air was filtered with HEPA (High efficiency particulate air) to produce a bioaerosol at 2 atmospheres. Place a diffusion dryer behind the aerosol generator (24 Jet Collison, BGI Collison) to remove moisture from the aerosol stream. $\quad \mathrm{A}^{210} \mathrm{Po}$ aerosol neutralizer was installed behind the diffusion dryer to remove the charge from the spores $^{[14]}$. The flow rate of the rotameter (Range of rotameter is 6-60 $\mathrm{mL} / \mathrm{min}$ ) is set to $16 \mathrm{~mL} / \mathrm{min}$. Finally, the PDMS layer of the microfluidic chip was removed to expose the collected spores, so the collected greenhouse crop airborne disease spores can be observed by the inverted microscope.

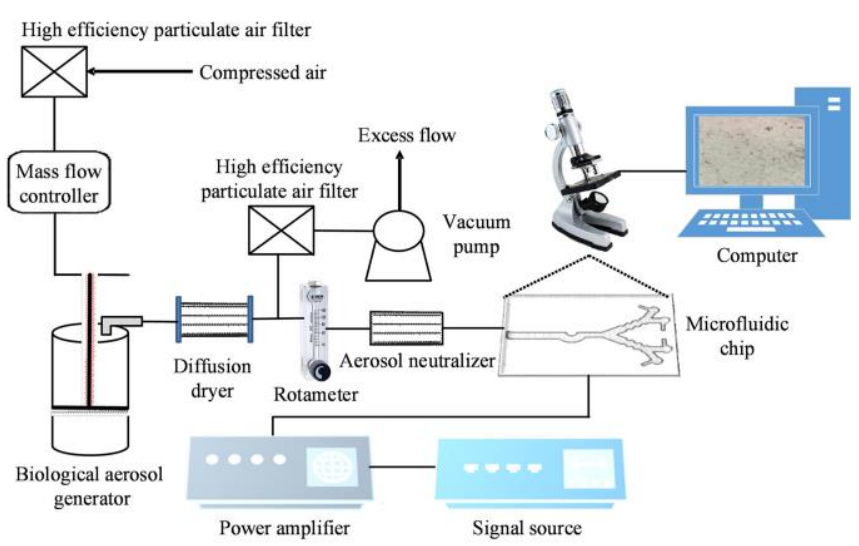

Figure 5 Schematic diagram of the experimental platform 
Figure 6 is an experimental image taken by the inverted microscope. $\quad B$. cinereal spores, $P$. cubensis spores and $P$. xanthii spores were collected in collection tank 1, collection tank 3 and collection tank 4, respectively. Therefore, the designed microfluidic chip can separate and collect greenhouse crop airborne disease spores.

The above experiments were carried out for $2 \mathrm{~min}$ and repeated five times. And the collected data were analyzed and summarized.
The results are shown in Table 2. As can be seen from Table 2, collection efficiency of $B$. cinereal spores, $P$. cubensis spores and $P$. xanthii spores were $75.7 \%, 83.8 \%$ and $89.4 \%$, respectively. Therefore, the proposed microfluidic chip can realize the greenhouse crops airborne disease spore separation and enrichment, which can provide a basis for the research of real-time monitoring technology for greenhouse airborne diseases.

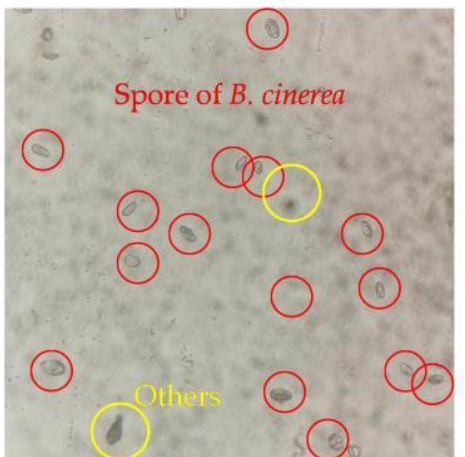

a. B. cinereal spores

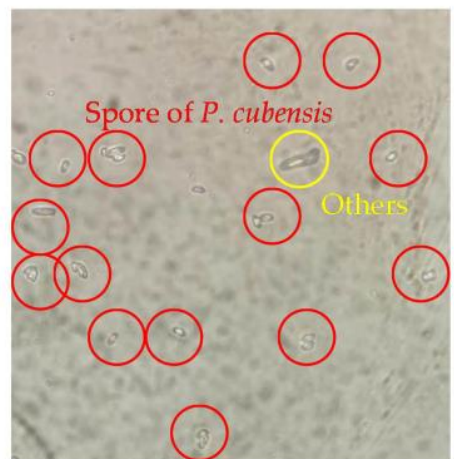

b. P. cubensis spores

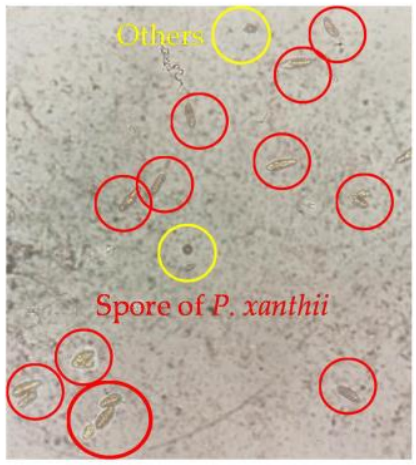

c. $P$. xanthii spores

Figure 6 Experimental results of spores separation and enrichment

Table 2 Statistics results of spore separation-enrichment

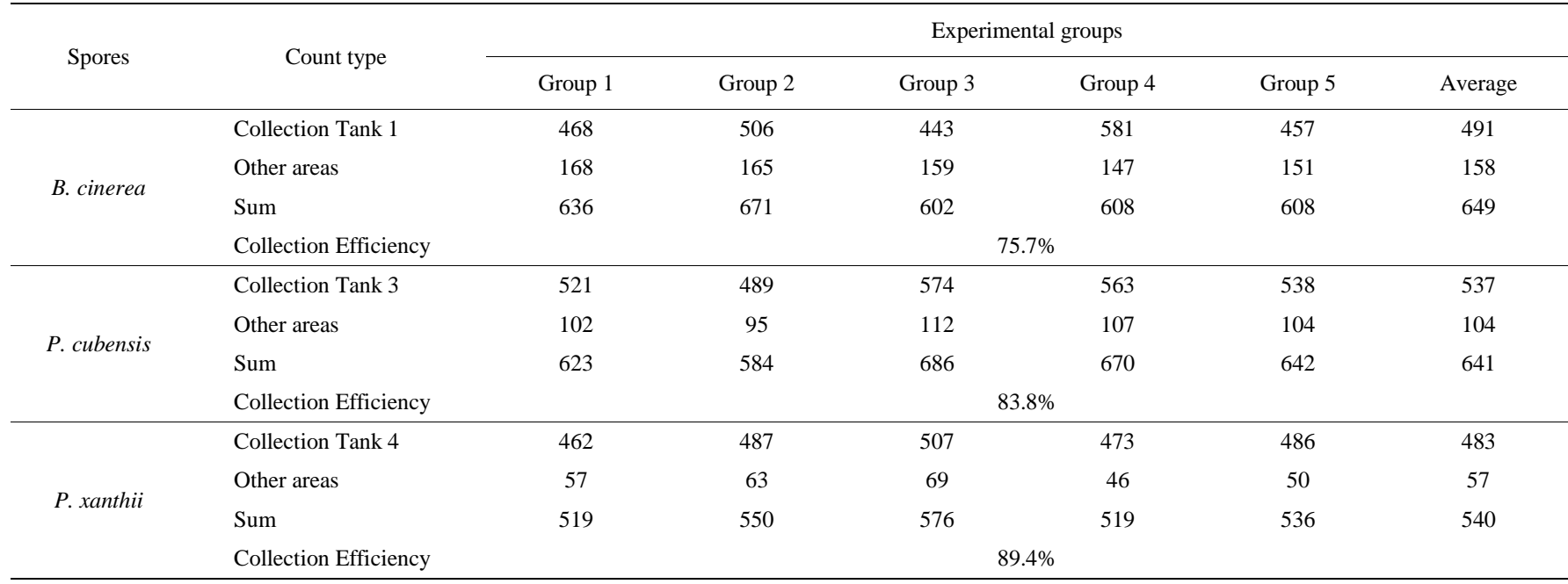

\section{Conclusions}

In this study, in order to realize early monitoring of crop diseases, a two-stage separation-enrichment structure microfluidic chip with a compound field for separation and enrichment the greenhouse crops airborne disease spores directly from gas flow was developed. The chip is mainly composed of three parts: arc structure pretreatment channel, semicircular electrode structure and collection tank. The microfluidic chip was simulated by using COMSOL 5.1 software. The simulation results showed that the separation and enrichment efficiency of $16 \mu \mathrm{m}$ particles, $25 \mu \mathrm{m}$ particles and $30 \mu \mathrm{m}$ particles was $88 \%, 91 \%$ and $94 \%$, respectively. The experimental verification results were observed under a microscope. The results showed that separation and enrichment efficiency of $B$. cinereal spores, $P$. cubensis spores and $P$. xanthii spores were $75.7 \%, 83.8 \%$ and $89.4 \%$, respectively.

\section{Acknowledgements}

This work was partially supported by the National Natural Science Foundation of China (Grant No. 32071905; No. 61771224).

\section{[References]}

[1] Wang T Y, Wu G X, Chen J W, Cui P, Chen Z X, Yan Y Y, et al Integration of solar technology to modern greenhouse in China: Current status, challenges and prospect. Renewable \& Sustainable Energy Reviews, 2017; 70: 1178-1188.

[2] Wang Y F, Ma G X, Du X X, Liu Y, Wang B, Xu G L, Mao H P. Effects of Nutrient Solution Irrigation Quantity and Downy Mildew Infection on Growth and Physiological Traits of Greenhouse Cucumber. Agronomy, 2020; 10(12): 1921. doi: 10.3390/agronomy10121921.

[3] Hafez Y M, Attia K A, Kamel S, Alamery S F, El-Gendy S, Al-Doss A A, et al. Bacillus subtilis as a bio-agent combined with nano molecules can control powdery mildew disease through histochemical and physiobiochemical changes in cucumber plants. Physiological and Molecular Plant Pathology, 2020; 111: 101489. doi: 10.1016/ j.pmpp.2020.101489.

[4] Tanaka K, Fukuda M, Amaki Y, Sakaguchi T, Inai K, Ishihara A, et al. Importance of prumycin produced by Bacillus amyloliquefaciens SD-32 in biocontrol against cucumber powdery mildew disease. Pest management science, 2017; 73: 2419-2428.

[5] Wallace E C, D'Arcangelo K N, Quesada-Ocampo L M. Population analyses reveal two host-adapted clades of Pseudoperonospora cubensis, the causal agent of cucurbit downy mildew, on commercial and wild cucurbits. Phytopathology, 2020; 110(9): 1578-1587.

[6] Akhmadeev A A, Salakhov M K. A new approach of recognition of ellipsoidal micro- and nanoparticles on AFM images and determination of 
their sizes. Measurement Science and Technology, 2016; 27(10): 105402. doi: 10.1088/0957-0233/27/10/105402.

[7] Lei Y, Yao Z F, He D J. Automatic detection and counting of urediniospores of Puccinia striiformis f. sp. tritici using spore traps and image processing. Scientific reports, 2018; 8:13647. doi: 10.1038/ s41598-018-31899-0.

[8] Chan B D, Icoz K, Huang W F, Chang C L, Savran C A. On-demand weighing of single dry biological particles over a 5-order-of-magnitude dynamic range. Lab on a Chip, 2014; 14(21): 4188-4196.

[9] Sireesha Y, Velazhahan R. Rapid and specific detection of Peronosclerospora sorghi in maize seeds by conventional and real-time PCR. European Journal of Plant Pathology, 2018; 150(2): 521-526.

[10] Bandamaravuri K B, Nayak A K, Bandamaravuri A S, Samad A. Simultaneous detection of downy mildew and powdery mildew pathogens on Cucumis sativus and other cucurbits using duplex-qPCR and HRM analysis. AMB Express, 2020; 10(1): 135. doi: 10.1186/s13568-02001071-x.

[11] Wada M, Tsukada M, Namiki N, Szymanski W W, Noda N, Makino H, et al. A two-stage virtual impactor for in-stack sampling of PM2.5 and PM10 in flue gas of stationary sources. Aerosol and Air Quality Research, 2016; 16(1): 36-45.

[12] Djoumi L, Vanotti M, Blondeau-Patissier V. Real time cascade impactor based on surface acoustic wave delay lines for PM10 and PM2.5 mass concentration measurement. Sensors, 2018; 18(1): 255. doi: 10.3390/ s18010255.

[13] Siani O Z, Targhi M Z, Sojoodi M, Movahedin M. Dielectrophoretic separation of monocytes from cancer cells in a microfluidic chip using electrode pitch optimization. Bioprocess and Biosystems Engineering, 2020; 43(9):1573-1586.

[14] Wang P, Yuan S Q, Yang N, Wang A Y, Fordjour A, Chen S B. The Collection method for crop fungal spores based on an efficient microfluidic device. Aerosol and Air Quality Research, 2020; 20(1): $72-79$.

[15] Yang N, Chen C Y, Li T, Li Z, Zou L R, Zhang R B, et al. Portable rice disease spores capture and detection method using diffraction fingerprints on microfluidic chip. Micromachines, 2019; 10(5): 289. doi: 10.3390/ mi10050289.

[16] Ren Q L, Liang C X, Wang Z X, Qu Z G. Continuous trapping of bacteria in non-Newtonian blood flow using negative dielectrophoresis with quadrupole electrodes. Journal of Physics D-applied Physics, 2021; 54(1): 015401. doi: 10.1088/1361-6463/abb726.

[17] Abd Rahman N, Ibrahim F, Yafouz B. Dielectrophoresis for biomedical sciences applications: A review. Sensors, 2017; 17(3): 449. doi: 10.3390/s17030449.

[18] Ettehad H M, Zarrin P S, Holzel R, Wenger C. Dielectrophoretic immobilization of yeast cells using CMOS integrated microfluidics. micromachines, 2020; 11(5): 501. doi: 10.3390/mi11050501.

[19] Zhang Y L, Chen X Y. Blood cells separation microfluidic chip based on dielectrophoretic force. Journal of the Brazilian Society of Mechanical Sciences and Engineering, 2020; 42(4): 206. doi: 10.1007/s40430-02002284-8.

[20] Zhang Y, Wang S Y, Chen J, Yang F, Li G Y. Separation of macrophages using a dielectrophoresis-based microfluidic device. Biochip Journal, 2020; 14(2): 185-194.

[21] Lee K, Lee J, Ha D, Kim M, Kim T. Low-electric-potential-assisted diffusiophoresis for continuous separation of nanoparticles on a chip. Lab on a Chip, 2020; 20(15): 2735-2747.

[22] Tajik P, Saidi M S, Kashaninejad N, Nguyen N T. Simple, cost-effective, and continuous 3D dielectrophoretic microchip for concentration and separation of bioparticles. Industrial \& Engineering Chemistry Research, 2020; 59(9): 3772-3783.

[23] Natu R, Martinez-Duarte R. Numerical model of streaming DEP for stem cell sorting. Micromachines, 2016; 7(12): 217. doi: 10.3390/mi7120217.

[24] Ayala-Mar S, Perez-Gonzalez V H, Mata-Gomez M A, Gallo-Villanueva R C, Gonzalez-Valdez J. Electrokinetically driven exosome separation and concentration using dielectrophoretic-enhanced PDMS-based microfluidics. Analytical Chemistry, 2019; 91(23): 14975-14982.

[25] Hirota Y, Hakoda M, Wakizaka Y. Separation characteristics of animal cells using a dielectrophoretic filter. Bioprocess and Biosystems Engineering, 2010; 33(5): 607-612.

[26] Han S I, Huang C, Han A. In-droplet cell separation based on bipolar dielectrophoretic response to facilitate cellular droplet assays. Lab on a Chip, 2020; 20(20): 3832-3841.

[27] Gascoyne P R C, Shim S, Noshari J, Becker F F, Stemke-Hale K. Correlations between the dielectric properties and exterior morphology of cells revealed by dielectrophoretic field-flow fractionation. Electrophoresis, 2013; 34(7): 1042-1050.

[28] Chen L, Liu X, Zheng X L, Zhang X L, Yang J, Tian T, et al. Dielectrophoretic separation of particles using microfluidic chip with composite three-dimensional electrode. Micromachines, 2020; 11(7): 700 doi: $10.3390 / \mathrm{mi} 11070700$.

[29] Alnaimat F, Mathew B, Hilal-Alnaqbi A. Modeling a dielectrophoretic microfluidic device with vertical interdigitated transducer electrodes for separation of microparticles based on size. Micromachines, 2020; 11(6): 563. doi: 10.3390/mi11060563.

[30] Zhang Z L, Luo Y, Nie X F, Yu D L, Xing X X. A one-step molded microfluidic chip featuring a two-layer silver-PDMS microelectrode for dielectrophoretic cell separation. Analyst, 2020; 145(16): 5603-5614.

[31] Wang Y F, Du X X, Ma G X, Liu Y, Wang B, Mao H P. Classification methods for airborne disease spores from greenhouse crops based on multifeature fusion. Applied Science, 2020; 10(21): 7850. doi: 10.3390/ app10217850.

[32] Zhu Y D, Zhang J Y, Li M Y, Zhao L J, Ren H R, Yan L G, et al. Rapid determination of spore germinability of Clostridium perfringens based on microscopic hyperspectral imaging technology and chemometrics. Journal of Food Engineering, 2020; 280: 109896. doi: 10.1016/ j.foodeng.2019.109896.

[33] Xu P F, Zhang R B, Yang N, Oppong P K, Sun J. High-precision extraction and concentration detection of airborne disease microorganisms based on microfluidic chip. Biomicrofluidics, 2019; 13(2): 024110. doi: 10.1063/1.5086087.

[34] Tian E Z, Xia F X, Wu J D, Zhang Y P, Li J, Wang H, et al. Electrostatic air filtration by multifunctional dielectric heterocaking filters with ultralow pressure drop. Acs Applied Materials \& Interfaces, 2020; 12(26): 29383-29392.

[35] Shi L Y, Shi X M, Zhou T, Liu Z Y, Liu Z Y, Joo S. A full-scale computational study on the electrodynamics of a rigid particle in an optically induced dielectrophoresis chip. Modern Physics Letters B, 2020; 34(22): 2050233. doi: 10.1142/S0217984920502334.

[36] Ho M T, Li J, Su W, Wu L, Borg M K, Li Z H, et al. Rarefied flow separation in microchannel with bends. Journal of Fluid Mechanics, 2020; 901: A26. doi: 10.1017/jfm.2020.585. 